\title{
Galois and Post Algebras of Compositions (Superpositions)
}

\author{
Maydim Malkov \\ Russian Research Center for Artificial Intelligence, Moscow, Russia
}

\section{Email address:}

mamalkov@gmail.com

\section{To cite this article:}

Maydim Malkov. Galois and Post Algebras of Compositions (Superpositions). Pure and Applied Mathematics Journal. Vol. 6, No. 4, 2017, pp. 114-119. doi: 10.11648/j.pamj.20170604.12

Received: June 10, 2017; Accepted: June 22, 2017; Published: July 20, 2017

\begin{abstract}
The Galois algebra and the universal Post algebra of compositions are constructed. The universe of the Galois algebra contains relations, both discrete and continuous. The found proofs of Galois connections are shorter and simpler. It is noted that anti-isomorphism of the two algebras of functions and of relations allows to transfer the results of the modern algebra of functions to the algebra of relations, and vice versa, to transfer the results of the modern algebra of relations to the algebra of functions. A new Post algebra is constructed by using pre-iterative algebra and by adding relations as one more universe of the algebra. The universes of relations and functions are discrete or continuous. It is proved that the Post algebra of relations and the Galois algebra are equal. This allows to replace the operation of conjunction by the operation of substitution and to exclude the operation of exist quantifier.
\end{abstract}

Keywords: Function Algebra, Relation Algebra, Universal Post Algebra

\section{Introduction}

The Post algebra was created by E. Post in [1] (1941). He used this algebra to construct the classification of Boolean functions.

The term "Post algebra" appeared in the first in [2] (1942). But the mathematically precise definition of this algebra was given first by A. Mal'cev in [3] (1976). Mal'cev gave definitions of two algebras, which he called preiterative and iterative Post algebras. The Post algebra is the pre-iterative algebra. The iterative algebra was created by S. Jablonskij ([4], 1958). He used it to construct a partial classification of finite-valued discrete functions. Therefore, he obtained results different from Post's results at constructing the classification of Boolean functions ([5], 1966).

The term "Galois connections" was introduced by G. Birkhoff ([6], 1940).

The Galois algebra of compositions was created by D. Geiger in [7] (1968) and by V. Bondarchuk, L. Kaluzhnin, V. Kotov, B. Romov in [8] (1969). A mathematically precise definition of the Galois algebra was given by S. Marchenkov in [9] (2000).

The term "Galois algebra of superpositions" is introduced in this article. This term is given because this algebra was used in [6 - 9] to construct the Galois connections.
Classification of all subalgebras of Post algebra was given for any $k$ in [10] (2014).

Many statements are given without proofs or with ideas of proofs. Proofs are omitted, if they are obvious. The formalization of the remaining proofs would lead to increase the article volume and to difficulty in understanding it. Some statements and its proofs are better formalized.

\section{Notation}

The $a$ symbol is used for variable values, the $\vec{a}$ character is used for a column that contains some variable values. The $i$ symbol is used to index an arbitrary element of a set or a component of a sequence. The symbol $n$ is used for arity of functions and relations. The symbols $f$ and $F$ are used for functions and for function sets, the symbols $r$ and $R$ are used for relations and for relation sets. The $S$ character is used for sorts of values of variables.

\section{Terminology}

Predicate is a logical formula, relation is an interpretation of a predicate by a table or matrix. Matrix lines are columns of a table, matrix columns are lines of a table. These matrices are used to preserve a relation by a function ([11]): $f\left(\breve{a}_{1}, \ldots, \breve{a}_{n}\right)=\breve{a}_{j}$, where $\breve{a}_{i}$ is any column (including equal) of a matrix, $\breve{a}_{j}$ is one of the columns of a matrix.

For simplicity, the term "relation table" is used instead of the term "relation table interpreting a predicate". Very often 
the terms "predicate" and "relation" are identified.

The set of values of a variable is called sort. For simplicity, all variables of relations and functions have the same sort. So a set of relations or functions has a sort, if these relations or functions have the same sort.

Relations and functions can be either discrete or continuous. They can be tabulated. A table of a continuous function is a discrete table which strings are presented by the lines of nodal values of variables such that the polynomial interpolation of values between nodes is highly accurate ${ }^{1}$. This excludes discontinuous functions. However, the result obtained for continuous functions are valid for discontinuous functions too. The relations can be continuous or partially continuous, if the set of continuous parts is finite or countable.

\section{Galois Algebra}

Definition 1. The Galois algebra $\mathrm{R}_{\mathrm{s}}$ is

$$
\mathrm{R}_{\mathrm{s}}=\left(R_{S} ; \zeta, \tau, \Delta, \exists, \&\right)
$$

where $R_{S}$ is a universe of all relations of a sort $S$ and $\zeta$, $\tau, \Delta, \exists, \&$ are fundamental (or $\Omega$ ) operations of the algebra.

The sort is: $\mathrm{N}_{\mathrm{k}}$ (the first $k$ natural numbers), $\mathrm{N}$ (natural numbers), $\mathrm{Z}$ (integers), $\mathrm{Q}$ (rational numbers), $\mathrm{R}$ (real numbers), $\mathrm{C}$ (complex numbers). It is generally accepted to denote $\mathrm{N}_{\mathrm{k}}$ by $k$ in this definition.

The members of the set $R_{S}$ are relations and they are denoted by $r$.

The fundamental operations are given over the set $R_{S}$. The operations $\zeta$ and $\tau$ permute variables in relations. The operation $\Delta$ reduces the number of variables by identifying the first two variables. The operation $\exists$ is an existence quantifier of the first variable. The operation $\&$ is a conjunction of two relations.

A. Mal'cev ([3]) gave the definitions of the first three operations for functions. But they are applicable to relations without changes.

The operation $\exists$ is $\exists x_{1} r\left(x_{1}, \ldots, x_{n}\right)$. As a result, the first column is removed from a table of a relation.

A function can be represented by a relation and its first column can be removed by $\exists$. But the result may not be a function. So the operation $\exists$ can be applied only to relations.

The conjunction of two relations $r_{1}^{n_{1}}$ and $r_{2}^{n_{2}}$ is

$$
r_{1}^{n_{1}}\left(x_{1}, \ldots, x_{n_{1}}\right) \wedge r_{2}^{n_{2}}\left(x_{n_{1}+1}, \ldots, x_{n_{1}+n_{2}}\right)
$$

where the variables $x_{1}, \ldots, x_{n_{1}}$ have not equal and variables

\footnotetext{
${ }^{1}$ For example, the table of real function $x_{1}+\ldots+x_{n}$ is a table of integer discreet function $x_{1}+\ldots+x_{n}$. The real function inherits all properties of the discreet function: it belongs to maximal clone preserving 0 and to monotone clone, the clone of these functions is non-fictitious ([11]) since it has one-membered basis $\left\{x_{1}+x_{2}\right\}$, and so on.
}

$x_{n_{1}+1}, \ldots, x_{n_{1}+n_{2}}$ have not equal. But some variables from $x_{1}, \ldots, x_{n_{1}}$ can be equal to some variables from $x_{n_{1}+1}, \ldots, x_{n_{1}+n_{2}}$. In this case, the conjunction of relations is constructed at first with unequal variables, and then the equality of the variables is realized.

This conjunction is possible only after adding the fictitious variables $x_{n_{1}+1}, \ldots, x_{n_{1}+n_{2}}$ to the relation $r_{1}$ and fictitious variables $x_{1}, \ldots, x_{n_{1}}$ to the relation $r_{2}$. After these additions and after consequent conjunction, the relation of arity $n_{1}+n_{2}$ is obtained. The number of lines in this relation is $n_{1} n_{2}$, and some of these lines can be equal. The first $n_{1}$ components of each line in $r_{1} \& r_{2}$ are one of lines of $r_{1}$, the following $n_{2}$ components are one of lines of $r_{2}$.

Fictitious variable is an $x_{j}$ for which

$$
\forall x_{j}^{\prime} \forall x_{j}^{\prime \prime} r\left(x_{1}, \ldots, x_{j-1}, x_{j}^{\prime}, x_{j+1}, \ldots, x_{n}\right) \equiv r\left(x_{1}, \ldots, x_{j-1}, x_{j}^{\prime \prime}, x_{j+1}, \ldots, x_{n}\right)
$$

If $n=1$ then there is a line with any other value $x_{j}^{\prime \prime}$ for every line with value $x_{j}^{\prime}$, i.e., the relation contains all values of its sort.

A fictitious variable can be added to an arbitrary relation by conjunction of this relation and the one-ary diagonal $\delta^{1}$ (this means that operation of adding fictitious variable is not primitive, i.e., the operation is not elementary).

A diagonal of a relation $r^{n}$ contains only $r^{n}(x, \ldots, x)$. The diagonal of the complete relation (the diagonal for the identically true $n$-ary predicate) is denoted by $\delta^{n}$. The diagonal of the empty relation (the diagonal for the identically false predicate) is denoted by $\delta^{0}$. The diagonal $\delta^{1}$, and only it, has a fictitious variable. The other complete diagonals have the same columns. The matrix of complete diagonals contains only one line, since all lines are equal. This line contains all values of a sort. Consequently, any function preserves all diagonals.

Ultra-diagonal ${ }^{2}$ is any diagonal $\delta^{n}$ or is conjunction of two or more complete diagonals including equal.

Composition (superposition) is the application of the fundamental operations to functions or relations.

The number of all possible operations over the set $R_{S}$ is infinite, but all of them are constructed from the fundamental operations. Therefore, the fundamental operations are primitive (they cannot be constructed but they can construct all other operations of an algebra). It was shown above that the operation of adding a fictitious variable to a relation is not primitive.

The fundamental operations are used to create universes that are subsets of the universe $R_{S}$.

Definition 2. Let $R_{1}$ be a subset of $R_{S}$. Universe of $R_{1}$ is

\footnotetext{
${ }^{2}$ Ultra diagonals are called diagonals in $[8,9,11]$. Ultra-diagonals are redundant, they are present only in the classification of coclones as a minimal coclone.
} 
the set $\left[R_{1}\right]$ containing

- members of $R_{1}$,

- the result of applying the operations of permutation, identification and existence to members of $\left[R_{1}\right]$,

- the result of conjunctions of members of $\left[R_{1}\right]$ :

$$
\begin{aligned}
& {\left[R_{1}\right]=R_{2} \Leftrightarrow }\left(\forall g \in R_{1} \quad g \in R_{2}\right) \wedge \\
&\left(\forall g \in R_{2} \quad \zeta g \in R_{2} \wedge \tau g \in R_{2} \wedge \Delta g \in R_{2} \wedge \exists g \in R_{2}\right) \wedge \\
& \wedge \forall g_{1}, g_{2} \in R_{2} \quad g_{1} \& g_{2} \in R_{2}
\end{aligned}
$$

This definition is iterative. At the first step of the iteration, $R_{2}$ contains all members of $R_{1}$. At the second step, compositions of members of $R_{2}$ are added to $R_{2}$. At the next step, the resulting set $R_{2}$ is added by compositions of its members. And so on.

A universe of functions is called a clone, if it contains the selective (projective) function $e_{1}^{2}\left(x_{1}, x_{2}\right)=x_{1}$. A clone also contains all selective functions $e_{m}^{n}\left(x_{1}, \ldots, x_{n}\right)=x_{m}$. A universe of relations is called a coclone, if it contains the diagonal $\delta^{2}$. Moreover, the coclone contains all the diagonals of $\delta^{n}$, since $\delta^{n+1}\left(x_{1}, \ldots, x_{n+1}\right)=\delta^{n}\left(x_{1}, \ldots, x_{n}\right) \wedge \delta^{2}\left(x_{n}, x_{n+1}\right)$. A coclone also contains all ultra-diagonals.

By the next lemmas, a set of functions preserving a relation is a clone, and a set of relations preserved by a function, is a coclone. The proof of the lemmas is given precise mathematically by using results of Mal'cev ([3]).

Lemma 1. A set $F=\operatorname{Pol}(r)$ is a clone for any relation $r$.

Proof. A set $\operatorname{Pol}(r)$ contains all selective functions $e_{m}^{n}$ since $e_{m}^{n}\left(\breve{a}_{1}, \ldots, \breve{a}_{n}\right)=\breve{a}_{m}$, where $\breve{a}_{i}$ is any column from the matrix of $r$.

Further it is proven that the set $\operatorname{Pol}(r)$ is a universe.

Let $f \in \operatorname{Pol}(r)$. Then $f$ preserves a relation $r$ : $f\left(\breve{a}_{1}, \ldots, \breve{a}_{n}\right)=\breve{a}_{j}$, where $\breve{a}_{i}(1 \leq i \leq n)$ are any columns of a matrix of $r, \breve{a}_{j}$ is one of the columns of this matrix.

If columns $\breve{a}_{i_{1}}$ and $\breve{a}_{i_{2}}$ of $f\left(\breve{a}_{1}, \ldots, \breve{a}_{n}\right)$ are permuted then there exists $1 \leq j_{0} \leq n$ such that

$$
f\left(\breve{a}_{1}, \ldots, \breve{a}_{i_{1}-1}, \breve{a}_{i_{2}}, \breve{a}_{i_{1}+1}, \ldots, \breve{a}_{i_{2}-1}, \breve{a}_{i_{1}}, \breve{a}_{i_{2}+1}, \ldots, \breve{a}_{n}\right)=\breve{a}_{j_{0}}
$$

since $f$ preserves $r$.

Therefore, the result of the operations $\zeta$ and $\tau$ over functions from $\operatorname{Pol}(r)$ is a function of $\operatorname{Pol}(r)$.

If the first two variables in a function $f$ are identifies then lines with unequal values of these variables will be removed from the table of $f$. The following lines will remain:

$$
f\left(\breve{a}_{1}^{\prime}, \breve{a}_{1}^{\prime}, \breve{a}_{3}^{\prime}, \ldots, \breve{a}_{n}^{\prime}\right)
$$

where $\breve{a}_{i}^{\prime}$ is $\breve{a}_{i}$ without some lines. But the function $f$ continues to preserve $r$ after removal of some lines in the matrix of $r$. Therefore, the result of the operation $\Delta$ is a function of $\operatorname{Pol}(r)$. It was necessary to prove that the result of the operation * over functions in $\operatorname{Pol}(r)$ is a function from $\operatorname{Pol}(r)$.

Let arbitrary functions $f_{1}$ and $f_{2}$ preserve the relation $r$ : $f_{1}\left(\breve{a}_{1}^{\prime}, \ldots, \breve{a}_{n_{1}}^{\prime}\right)=\breve{a}_{j_{1}}^{\prime}, f_{2}\left(\breve{a}_{1}^{\prime \prime}, \ldots, \breve{a}_{n_{2}}^{\prime \prime}\right)=\breve{a}_{j_{2}}^{\prime \prime}$, where $\breve{a}_{i}^{\prime}$ and $\breve{a}_{i}^{\prime \prime}$ are any columns from the matrix of $r, \vec{a}_{j_{1}}^{\prime}$ and $\breve{a}_{j_{2}}^{\prime \prime}$ are one of the columns of this matrix. Then

$$
f_{1}\left(f_{2}\left(\breve{a}_{1}^{\prime \prime}, \ldots, \breve{a}_{n_{2}}^{\prime \prime}\right), \breve{a}_{2}^{\prime}, \ldots, \breve{a}_{n_{1}}^{\prime}\right)=f_{1}\left(\breve{a}_{j_{2}}^{\prime \prime}, \breve{a}_{2}^{\prime}, \ldots, \breve{a}_{n_{1}}^{\prime}\right)
$$

and there exists $j_{3}$ such that $f_{1}\left(\vec{a}_{j_{2}}^{\prime \prime}, \vec{a}_{2}^{\prime}, \ldots, \vec{a}_{n_{1}}^{\prime}\right)=\breve{a}_{j_{3}}^{\prime}$.

Lemma 2. A set $R=\operatorname{Inv}(f)$ is a coclone for any function $f$.

Proof. The set $\operatorname{Inv}(f)$ contains all diagonals $\delta^{n}$, since any function preserves any diagonal.

Further it is proven that the set $\operatorname{Inv}(f)$ is a universe.

A function $f$ preserving some relation will preserve the relation after permutation of columns in the table of this relation, i.e., after permutation of lines in the matrix of the relation.

Therefore, the result of the operations $\zeta$ and $\tau$ over relations from $\operatorname{Inv}(f)$ is a relation from $\operatorname{Inv}(f)$.

If two variables in $r$ are identified then lines with unequal values of the variables will be removed from the table of $r$. Therefore, corresponding columns in the matrix of $r$ will be removed. But the remained columns have two lines to be equal The removed columns have not these two lines to be equal. So no of these columns can be values of the function when remained columns are values of variables of the function. Therefore, the result of the operation $\Delta$ over relations from $\operatorname{Inv}(f)$ is a relation from $\operatorname{Inv}(f)$.

The result of the operation $\exists$ over relations from $\operatorname{Inv}(f)$ is a relation from $\operatorname{Inv}(f)$ too. Indeed, removing a column in a relation table is removing a line in the relation matrix. But a function preserving a relation will preserve the relation after removing one or more lines.

It is needed to prove finally that the result of the operation \& over relations from $\operatorname{Inv}(f)$ is a relation from $\operatorname{Inv}(f)$.

Let $r^{n_{1}+n_{2}}=r_{1}^{n_{1}} \& r_{2}^{n_{2}}$ and let $r_{1}^{n_{1}}$ and $r_{2}^{n_{2}}$ be relations preserved by a function $f: f\left(\breve{a}_{1}^{\prime}, \ldots, \breve{a}_{n}^{\prime}\right)=\breve{a}_{J_{1}}^{\prime}$, $f\left(\breve{a}_{1}^{\prime \prime}, \ldots, \breve{a}_{n}^{\prime \prime}\right)=\breve{a}_{j_{2}}^{\prime \prime}$, where $\breve{a}_{i}^{\prime}$ and $\breve{a}_{i}^{\prime \prime}$ are any columns from matrices of $r_{1}^{n_{1}}$ and of $r_{2}^{n_{2}}$, and where $\breve{a}_{j_{1}}^{\prime}, \vec{a}_{j_{2}}^{\prime \prime}$ are some columns of these matrices.

A table of a relation $r$ contains the first $n_{1}$ columns, each line of which is a line from the table $r_{1}$. And the table contains the other $n_{2}$ columns, each line of which is a line from the table $r_{2}$. Hence, the matrix of the relation $r$ contains 
columns which first $n_{1}$ lines are lines from the matrix of $r_{1}$ and the other $n_{2}$ lines are lines from the matrix of $r_{2}$. Hence, a function $f$ preserving $r_{1}$ and $r_{2}$ preserves $r_{1} \& r_{2}$ too. $\square$

\section{Galois Connections}

The set of all functions preserving the relation $r$ is $\operatorname{Pol}(r)$. The set of all relations preserved by a function $f$ is $\operatorname{Inv}(f)$, and

$$
\operatorname{Pol}(R)=\bigcap_{r \in R} \operatorname{Pol}(r), \quad \operatorname{Inv}(F)=\bigcap_{f \in F} \operatorname{Inv}(f)
$$

for the set of relations $R$ and for the set of functions $F$. The Galois connection between $\operatorname{Pol}(R)$ and $\operatorname{Inv}(F)$ is given by two theorems, much simpler proofs of which are given below.

The first Galois connection is given by the following theorem.

Theorem 1. If and only if a set $F$ is a clone then $F=\operatorname{Pol}(\operatorname{Inv}(F))$.

Proof. If $F=\operatorname{Pol}(\operatorname{Inv}(F))$ then $F$ is a clone (lemma 1).

Let $F$ be a clone.

Let $R_{0}$ be the set of all $r$ preserved by all $f \in F$ : $R_{0}=\operatorname{Inv}(F)$. And let $r_{i} \in R_{0}$. Then $\operatorname{Pol}\left(r_{i}\right)$ contains all functions of $F$ and functions that do not preserve the set of the other relations. But then the intersection of all these functions is $F$, i.e., $F=\operatorname{Pol}(\operatorname{Inv}(F))$.

The second Galois connection is contained in one more theorem.

Theorem 2. If and only if an $R$ is a coclone then $R=\operatorname{Inv}(\operatorname{Pol}(R))$.

Proof. If $R=\operatorname{Inv}(\operatorname{Pol}(R))$ then $R$ is a coclone (lemma 2). Let $R$ be a coclone.

Let $F_{0}$ be the set of all $f$ that preserve all $r \in R$ : $F_{0}=\operatorname{Pol}(R)$. And let $f_{i} \in F_{0}$. Then $\operatorname{Inv}\left(f_{i}\right)$ contains all relations from $R$ and relations that are not preserved by the set of other functions. But then the intersection of all these relations is $R$, i.e., $R=\operatorname{Inv}(\operatorname{Pol}(R))$.

The result of these two theorems is a very important

Theorem 3. Diagrams of clone inclusions and of coclones inclusions are anti-isomorphic:

$$
R_{1} \subset R_{2} \rightarrow \operatorname{Pol}\left(R_{2}\right) \subset \operatorname{Pol}\left(R_{1}\right), \quad F_{1} \subset F_{2} \rightarrow \operatorname{Inv}\left(F_{2}\right) \subset \operatorname{Inv}\left(F_{1}\right)
$$

Proof. There is a one-to-one connection between clones and coclones. To each clone $F$ there corresponds a coclone $R=\operatorname{InvF}$, and to each coclone $R$ there corresponds the same clone $F=\operatorname{Pol}(R)=\operatorname{Pol}(\operatorname{Inv}(F) \quad($ theorem 1). Likewise, to each coclone $R$ there corresponds a clone $F=P o l R$, and to each clone $F$ there corresponds the same coclone $R=\operatorname{Inv}(F)=\operatorname{Inv}(\operatorname{Pol}(R)$ (theorem 2$)$.

Additional relations in the coclone $R_{2}$ reduce the number of functions in the clone $F_{2}=\operatorname{Pol}\left(R_{2}\right)$ with respect to the clone $F_{1}=\operatorname{Pol}\left(R_{1}\right)$. Indeed, $\operatorname{Pol}\left(R_{2}\right)=\bigcap_{r \in R_{2}} \operatorname{Pol}(r)$, and additional $r$ reduce $F_{2}=\operatorname{Pol}\left(R_{2}\right)$ at intersection. So $R_{1} \subset R_{2} \rightarrow \operatorname{Pol}\left(R_{2}\right) \subset \operatorname{Pol}\left(R_{1}\right)$.

Likewise, additional functions in a clone $F_{2}$ reduce the number of relations in the coclone $R_{2}=\operatorname{Inv}\left(F_{2}\right)$ with respect to the coclone $R_{1}=\operatorname{Inv}\left(F_{1}\right)$, since $\operatorname{Inv}\left(F_{2}\right)=\bigcap_{f \in F_{2}} \operatorname{Inv}(f)$. And $F_{1} \subset F_{2} \rightarrow \operatorname{Inv}\left(F_{2}\right) \subset \operatorname{Inv}\left(F_{1}\right)$

The anti-isomorphism of clones and coclones generates an anti-isomorphism of the algebra of functions and of the algebra of relations.

The main objects of mathematics are functions and relations that are objects of the algebra of functions and the algebra of relations. The main problem of these algebras is a classification of their objects. This classification is realized by diagrams of inclusions of clones and coclones. To build a classification of clones and then to classify coclones is much easier than to do the reverse - to build a classification of coclones and then to classify clones.

The anti-isomorphism of these algebras allows to transfer the results of the modern algebra of functions to the algebra of relations, and to transfer the results of the modern algebra of relations to the algebra of functions.

\section{Post Algebra}

The definition of the Post algebra for discrete functions was given by Mal'cev ([3]). This definition is added by continuous functions and by discrete and continuous relations.

Definition 3. The Post Algebra $\mathrm{P}_{\mathrm{s}}$ is

$$
\mathrm{P}_{\mathrm{s}}=\left(P_{S} ; \zeta, \tau, \Delta, *\right)
$$

where $P_{S}$ is a universe of the algebra, $S$ is a sort of members of the universe, $\zeta, \tau, \Delta$ and $*$ are fundamental operations of the algebra.

The universe can contain functions or relations: $P_{S}=F_{S}$ or $P_{S}=R_{S}$.

First three operations coincide with the first three operations of the Galois algebra. These operations are applicable to functions without changes.

Operation * for functions is substitution $f_{2}^{n_{2}}$ instead of the first variable in $f_{1}^{n_{1}}$ :

$$
\begin{aligned}
f_{1}^{n_{1} * f_{2} n_{2}} & =f_{3}^{n_{1}+n_{2}-1} \Leftrightarrow \forall x_{1}, \ldots, x_{n_{1}+n_{2}-1} \quad f_{3}^{n_{1}+n_{2}-1}\left(x_{1}, \ldots, x_{n_{1}+n_{2}-1}\right)= \\
& \left.=f_{1}^{n_{1}}\left(f_{2}^{n_{2}}\left(x_{1}, \ldots, x_{n_{2}}\right), x_{n_{2}+1}, \ldots, x_{n_{1}+n_{2}-1}\right)\right)
\end{aligned}
$$

Operation * for relations is substitution $r_{2}^{n_{2}}$ instead of the 
first variable in $r_{1}^{n}$ :

$$
r_{1}^{n_{1}} * r_{2}^{n_{2}} \Leftrightarrow \forall x_{1}, \ldots, x_{n_{1}+n_{2}-1} r_{1}\left(r_{2}\left(x_{1}, \ldots, x_{n_{2}}\right), x_{n_{2}+1}, \ldots, x_{n_{1}+n_{2}-1}\right)
$$

where

$$
r_{1}\left(r_{2}\left(x_{1}, \ldots, x_{n_{2}}\right), x_{n_{2}+1}, \ldots, x_{n_{1}+n_{2}-1}\right) \Leftrightarrow r_{2}\left(x_{1}, \ldots, x_{n_{2}}\right) \wedge r_{1}\left(x_{n_{2}}, \ldots, x_{n_{1}+n_{2}-1}\right)
$$

Substitution of the relation $r_{2}^{n_{2}}$ into the relation $r_{1}^{n_{1}}$ generates a relation of arity $n_{1}+n_{2}-1$. Substitution of a null-ary relation into an arbitrary relation reduces arity of this arbitrary relation by one. An exception is substitution of a null-ary relation into a null-ary relation. The result of this substitution is a null-ary relation.

Substitution of relations includes substitution of functions. Indeed, the substitution operation for three-ary functions is

$$
f\left(x_{1}, x_{2}, x_{3}\right)=f_{1}\left(f_{2}\left(x_{1}, x_{2}\right) x_{3}\right)
$$

But there is no information about the values of the functions $f, f_{1}$ and $f_{2}$. Including this information, $f\left(x_{1}, x_{2}, x_{3}\right)=x_{4} \equiv f_{1}\left(f_{2}\left(x_{1}, x_{2}\right)=x_{0}, x_{3}\right)=x_{4}$ is got.

The variable $x_{0}$ is a fictitious, since it is not present in the left part.

The same substitution operation for relations is

$$
r\left(x_{1}, x_{2}, x_{0}, x_{3}, x_{4}\right) \equiv r_{2}\left(x_{1}, x_{2}, x_{0}\right) \wedge r_{1}\left(x_{0}, x_{3}, x_{4}\right)
$$

Here functions $f_{1}$ and $f_{2}$ are represented by three-ary relations $r_{1}$ and $r_{2}$. The relation $r$ represents the function $f$ after removing the fictitious variable $x_{0}$.

The operation $\nabla$ (which adds a fictitious variable to a relation) is absent in the definition of the Post algebra. This operation is not primitive, since substitution a two-ary relation with two fictitious variables into any relation adds a fictitious variable to this relation.

There are subalgebras of the Post algebra. A universe of a subalgebra is closure of a subset of $P_{S}$. But fundamental operations are equal to fundamental operations of $P_{S}$.

\section{Galois and Post Algebras Are Equal}

The universes of the Galois algebra and of the Post algebra are equal for $P_{S}=R_{S}$. The fundamental operations $\zeta, \tau$, $\Delta$ are present in both algebras. The operations \&,$\exists$ and the operation are different.

Lemma 3. The operations \& and $\exists$ are generated by the primitives of the Post algebra.

Proof. First the theorem is proven for \&, and then for $\exists$.

- Let a conjunction be

$$
r_{1}^{n_{1}}\left(x_{1}, \ldots, x_{n_{1}}\right) \& r_{2}^{n_{2}}\left(x_{n_{1}+1}, \ldots, x_{n_{1}+n_{2}}\right)
$$

It is necessary to construct substitution $r_{3}^{n_{3}} * r_{4}^{n_{4}}$ equaling the conjunction above.

Let $n_{3}=n_{2}+1$, let $r_{3}^{n_{3}}$ be obtained from $r_{2}^{n_{2}}$ by adding a fictitious variable as the first. And let $n_{4}=n_{1}, r_{4}{ }^{n_{4}}=r_{1}^{n_{1}}$. Then

$$
\begin{aligned}
& r_{3}^{n_{3}}\left(r_{4}^{n_{4}}\left(x_{1}, \ldots, x_{n_{4}}\right), x_{n_{4}+1}, \ldots, x_{n_{3}+n_{4}}\right) \equiv \\
\equiv & r_{4}^{n_{4}}\left(x_{1}, \ldots, x_{n_{1}}\right) \wedge r_{3}^{n_{3}}\left(x_{n_{1}}, \ldots, x_{n_{1}+n_{2}+1}\right) \equiv \\
\equiv & r_{1}^{n_{1}}\left(x_{1}, \ldots, x_{n_{1}}\right) \& r_{2}^{n_{2}}\left(x_{n_{1}+1}, \ldots, x_{n_{1}+n_{2}}\right)
\end{aligned}
$$

Indeed, $r_{3}^{n_{3}}$ has the first variable to be fictitious before intersection. After intersection this first variable and the last variable of $r_{4}^{n_{4}}$ are identified, but identification of this fictitious variable and $x_{n_{4}}$ gives $x_{n_{4}}$. And this identification decreases the number of variables. The number equals $n_{1}+n_{2}$.

- Substitution of null-ary relation instead of the first variable in an arbitrary relation $r^{n}$ implies removal of the first variable, i.e., it leads to $\exists x_{1} r^{n}\left(x_{1}, \ldots, x_{n}\right)$.

Lemma 4 . The operation * is generated by primitives of the Galois algebra.

Proof. If the first variable in $r_{1}$ and the last variable in $r_{2}$ are identified in the conjunction of $r_{1} \& r_{2}$ then $r_{1} * r_{2}$ is got.

Hence, the sets of all possible operations in both algebras are equal.

Theorem 4. Post algebra of relations and Galois algebra are equal.

Proof follows from lemmas 3 and 4.

\section{Conclusion}

The Galois algebra of superpositions is constracted. The universe of the algebra contains both discrete and continuous relations. The fundamental operations of this algebra include the conjunction and the existence quantifier. Much shorter and simpler proofs of Galois connections is found (the proofs of Galois connections in the fundamental papers take several pages). It is noted that the anti-isomorphism of inclusions of clones and coclones allows a laborious classification of relations to replaced by a less laborious classification of functions. It is shown that the ultra-diagonals are redundant and can be replaced by more simpler diagonals.

The Post algebra has been constructed such that the algebra has universe containing either discrete or continuous functions or relations. Almost all researchers include the addition of fictitious variables to the Post algebra as one more fundamental operation. It is shown that this operation is not a primitive.

It is shown that the Galois algebra and the Post algebras of relations are equal. But the Post algebra is simpler and includes the algebra of functions too. 


\section{References}

[1] Post E. L., Two-valued iterative systems of mathematical logic. Princeton Univ. Press, Princeton (1941).

[2] Rosenbloom Paul C., Post algebras. I. Postulates and general theory, Amer. J. Math. 64, 167-188 (1942).

[3] Mal'cev A. I., Iterative Post algebras, NGU, Novosibirsk, (Russian) (1976).

[4] Jablonskij S. V., Functional constructions in many-valued logics (Russian). Tr. Mat. Inst. Steklova, 51 5-142 (1958).

[5] Jablonskij, S. W., Gavrilov, G. P., Kudryavcev, V. B., Boolean function and Post classes (Russian), Nauka, Moscow (1966).

[6] Birkgoff G., Lattice theory, American Math. Soc., Providence Rhode island (1940)

[7] Geiger D., Closed systems of functions and predicates Pacific J. Math. 27, 95--100 (1968).

[8] Bondarchuk, V. G., Kaluzhnin, L. A., Kotov, V. N., Romov, B. A., Galois theory for Post algebras I-II. (Russian) Kibernetika, 3, 1--10 (1969), 5, 1-9 (1969). English translation: Cybernetics, 243--252 and 531-539 (1969).

[9] Marchenkov C., Basic of Boolean functions theory (Russian), Fizmatlit, Moscow (2000).

[10] Malkov M. A., Classification of closed sets of functions in multi-valued logic. Sop transaction on applied math. 1:3 (2014).

[11] Lau D., Functions algebra on finite sets, Springer (2006). 